\title{
RENDIMENTO DO FEIJOEIRO IRRIGADO SUBMETIDO A DIFERENTES LÂMINAS DE ÁGUA COM IRRIGAÇÃO POR SULCO
}

\author{
Marcos Vinícius Folegatti', Vital Pedro da Silva Paz $^{2}$ \& Antonio Sanchez de Oliveira ${ }^{3}$
}

\begin{abstract}
RESUMO
O presente trabalho teve como objetivo analisar o rendimento do feijoeiro sob diferentes lâminas de água aplicadas com irrigação por sulco. Foi considerada uma declividade de $0,6 \%$ no sulco e adotadas as vazões de $0,35 \mathrm{~L} \mathrm{~s}^{-1}$ e $1,0 \mathrm{~L} \mathrm{~s}^{-1}$, sendo que esta última, após o avanço, foi reduzida para $0,5 \mathrm{~L} \mathrm{~s}^{-1}$. As lâminas de água aplicadas foram de 53,8,33,3 e 27,3 mm e o delineamento experimental foi o de blocos casualizados, com quatro tratamentos. Três tratamentos corresponderam, respectivamente, a 100, 50 e $0 \%$ de lâmina útil no final do sulco, enquanto o quarto tratamento serviu de testemunha e não recebeu irrigação. A aplicação de 50\% da lâmina de água útil no final da parcela não alterou significativamente a produtividade do feijoeiro. A diminuição da lâmina de água ao longo do sulco, resultou em menores valores dos parâmetros de rendimento, devido ao estresse gradual nos tratamentos de 0 e $50 \%$ da lâmina útil aplicada.
\end{abstract}

Palavras-chave: irrigação por sulco, feijoeiro, produção, Phaseolus vulgaris $\mathrm{L}$.

\section{YIELD OF FURROW IRRIGATED BEAN UNDER DIFFERENT WATER DEPTHS}

\begin{abstract}
The objective of this work was to study the yield of bean crop under different water depths applied by furrow irrigation. The slope of furrows was $0.6 \%$ and the water flow adjusted to 0.35 and $1.0 \mathrm{~L} \mathrm{~s}^{-1}$, the latter being reduced to $0.5 \mathrm{~L} \mathrm{~s}^{-1}$ after the water reached the end of the furrow. Mean water depths applied in each irrigation were of $53.80,33.30$ and $27.30 \mathrm{~mm}$, corresponding respectively to treatments of 100, 50 and $0 \%$ of the water depth applied at the end of the furrows. Another treatment did not receive water by irrigation and was used for comparison. The experimental design consisted of randomized blocks with four treatments and sub-plots. The production did not reduce significantly when $50 \%$ of the water depth was applied at the end of the furrow. The reduction of water along the furrow resulted in reduced production, from the beginning to the end of the furrow, due to gradual water stress applied on treatments of 50 and $0 \%$.
\end{abstract}

Key words: furrow irrigation, dry bean, Phaseolus vulgaris L.

\section{INTRODUÇÃO}

A baixa produtividade da cultura do feijoeiro tem decorrido principalmente da irregularidade ou deficiência das precipitações pluviométricas. Essa produtividade pode ser melhorada com a utilização do método de irrigação por sulco, dentre outros. Os elementos mais importantes a serem considerados neste método são a infiltração e a velocidade de avanço da água no sulco,

\footnotetext{
${ }^{1}$ Prof. Associado, Departamento de Engenharia Rural, ESALQ/USP. Av. Pádua Dias 11, CP 09, 13418 - 900, Piracicaba, SP. Fone: (019) 429 4217. E-mail: mvfolega@ carpa.ciagri.usp.br

${ }^{2}$ Prof. Visitante, Dr. em Irrigação e Drenagem, Departamento de Agronomia, Universidade Federal de Uberlândia. Av. Amazonas s/n. Bloco 2E, CP 593, 38400 - 902, Uberlândia, MG. Fone: (034) 2182225

${ }^{3}$ Prof. Titular, Departamento de Engenharia Rural, ESALQ/USP
} 
influenciadas pela vazão, pelo tipo de solo, pela declividade do terreno, pelo comprimento e espaçamento do sulco e pela duração da irrigação.

Segundo registros do Agrianual (1996) nos últimos anos no Brasil ocorreram aumentos na produtividade do feijão, citando a produtividade de $1000 \mathrm{~kg} \mathrm{ha}^{-1}$ como baixa, de $1600 \mathrm{~kg} \mathrm{ha}^{-1}$ como média e de $2300 \mathrm{~kg} \mathrm{ha}^{-1}$ como alta produtividade.

Bascur \& Fritsh (1975) estudaram diferentes situações de manejo do método de irrigação por sulco, ou seja, todos os sulcos irrigados simultaneamente, os irrigados alternadamente, sulcos irrigados simultaneamente mas com semeadura no talude e os sulcos irrigados para cada duas fileiras de planta e, adotando freqüências de irrigação correspondentes aos potenciais matriciais de $-40 \mathrm{kPa}$ e $-75 \mathrm{kPa}$, observaram que o número de vagens por planta e o número de grãos por vagem não foram afetados pelos métodos, pela frequiência nem por suas combinações, mas o peso de grãos aumentou de forma significativa com a freqüência de $40 \mathrm{kPa}$.

Millar \& Choudhury (1980) observaram que 80 a $90 \%$ do potencial de produção da cultura do feijoeiro são obtidos com um manejo de irrigação, no qual se fornece água ao solo quando o mesmo apresenta potencial matricial de -170 a $-17 \mathrm{kPa}$, respectivamente. Frizzone (1986) estudando o efeito da lâmina de água sobre o rendimento de grãos e componentes de produção do feijoeiro (Phaseolus vulgaris, $\mathrm{L}$ ) verificou que o máximo rendimento relativo de grãos foi obtido no regime de irrigação a - $19 \mathrm{kPa}$, correspondendo a uma freqüência de irrigação de aproximadamente 6 dias.

Garrido \& Teixeira (1978) estudando o efeito de diferentes níveis de umidade no solo sobre o rendimento do "feijão das águas", observaram que para o rendimento de grãos houve aumento significativo em relação à testemunha, no tratamento em que a irrigação era feita quando a umidade do solo atingia $70 \%$ da água disponível. Para o "feijão da seca" os autores observaram, ainda, que nos tratamentos irrigados aos níveis de 40, 60 e $80 \%$ da água disponível, ocorreu aumento significativo no rendimento de grãos em relação ao tratamento não irrigado. Para Guimarães (1988) a sensibilidade do feijoeiro ao déficit hídrico é devida à baixa capacidade de recuperação da planta após o déficit hídrico e ao seu sistema radicular pouco desenvolvido. $\mathrm{O}$ déficit ou o excesso de água sobre a cultura afeta os componentes de produção de maneira diferente, dependendo da intensidade, da duração e do estádio de desenvolvimento em que ocorre; já o excesso de água no solo provoca prejuízos à cultura devido à redução de oxigênio e diminuição da atividade microbiana do solo.

Fischer \& Weaver (1974) e Doorenbos \& Pruitt (1975) indicam, para o feijoeiro, os estádios do desenvolvimento críticos em relação ao déficit de água, como o preflorescimento, florescimento e enchimento de grãos, nos quais a falta de água causará redução no rendimento, no número de vagens por planta e no peso de grãos por vagem. Pozzebon (1995) estudando o comportamento do feijoeiro ao déficit hídrico no sul do Brasil, verificou que o número de vagens e o número de sementes por vagem foram afetados. Diversos estudos sobre o déficit hídrico na fase de floração confirmam a redução da produtividade (Magalhães et al., 1979; Hostalácio \& Válio, 1988).
O manejo adequado da irrigação constitui na escolha correta do método de aplicação de água e no estabelecimento de critérios para determinação da necessidade hídrica das culturas, resultando em níveis ótimos de produtividade.

O objetivo principal deste trabalho consistiu em se estudar o desenvolvimento do feijoeiro, sob diferentes lâminas de água aplicadas com irrigação por sulco.

\section{MATERIAL E MÉTODOS}

O experimento foi realizado na área experimental do Departamento de Engenharia Rural da Escola Superior de Agricultura "Luiz de Queiroz"/USP, Piracicaba, SP. O clima da região, segundo a classificação de Köppen, é do tipo Cwa-mesotérmico, subtropical úmido com estiagem de inverno, temperatura média do mês mais frio inferior a $18^{\circ} \mathrm{C}$ e a do mês mais quente superior a $22^{\circ} \mathrm{C}$, também denominado tropical de altitude.

O solo da área experimental foi classificado como Terra Roxa Estruturada. Para a instalação do experimento foram determinadas as características físicas e químicas do solo e a análise granulométrica realizada para as camadas de $0-0,20 \mathrm{~m}$ e de 0,20 - 0,40 m, indicou ser um solo de classe textural argila.

No experimento foram utilizados 95 sulcos com declividade média de $0,6 \%$, divididos em cinco blocos casualizados, cada bloco com quatro tratamentos, da seguinte forma: $\mathrm{T}_{1}-100 \% \mathrm{da}$ lâmina útil aplicada no final do sulco; $\mathrm{T}_{2}-50 \%$ da lâmina útil aplicada no final do sulco; $\mathrm{T}_{3}-0 \%$ da lâmina útil aplicada no final do sulco, e $\mathrm{T}_{4}$ - sem irrigação. A lâmina útil de irrigação é a altura de água a ser fornecida ao solo, até a profundidade efetiva do sistema radicular, compreendida entre a capacidade de campo e o ponto de murcha permanente.

Os tratamentos $\mathrm{T}_{1}$ e $\mathrm{T}_{2}$ foram conduzidos com a vazão de $1,0 \mathrm{~L} \mathrm{~s}^{-1}$ e o tratamento $\mathrm{T}_{3}$ com a vazão de $0,35 \mathrm{~L} \mathrm{~s}^{-1}$. Para os tratamentos $\mathrm{T}_{1}$ e $\mathrm{T}_{2}$ utilizou-se redução de vazão de $1,0 \mathrm{~L} \mathrm{~s}^{-1}$ para $0,5 \mathrm{~L} \mathrm{~s}^{-1}$ após a água ter alcançado o final do sulco, cujas parcelas consistiam de três sulcos consecutivos isolados por um sulco sem irrigação, sendo o sulco central utilizado para avaliação; os sulcos eram espaçados em $1,0 \mathrm{~m}$, com comprimento de $120 \mathrm{~m} \mathrm{e} 0,20 \mathrm{~m}$ de profundidade.

Para o suprimento de água foi utilizado um reservatório de nível constante com cota superior à da área experimental, possibilitando a condução da água por gravidade até a parcela, distribuindo-se a água nos sulcos por meio de sifões plásticos.

Conhecendo-se a água disponível no solo e as equações de avanço (Eq. 2) e infiltração (Eq. 3) da água nos sulcos, foram determinados os tempos de aplicação de água para 100 e 50\% da água disponível.

O tempo total de irrigação é dado pela expressão (Frizzone, 1992):

$$
T_{i}=T_{a p}+T_{a v}+T_{r}
$$

em que,

$\mathrm{T}_{\mathrm{i}} \quad$ - tempo total de irrigação ou de oportunidade de infiltração

$\mathrm{T}_{\mathrm{ap}}$ - tempo de aplicação ou de duração da fase de reposição

$\mathrm{T}_{\text {av }}^{\text {ap }}$ - tempo de avanço

$\mathrm{T}_{\mathrm{r}}$ - tempo de recessão. 
Foram instalados tensiômetros nas profundidades de 0,15 , 0,30 e 0,45 m numa das parcelas, para monitorar o potencial matricial da água no solo nas diversas fases da cultura e nos diferentes tratamentos. Para o manejo da irrigação foram utilizados dados de evaporação de um tanque Classe A, considerando-se o coeficiente do tanque (Kp) em função da umidade relativa do ar, velocidade do vento e bordadura do tanque, segundo Doorenbos \& Kassam (1979). O coeficiente de cultura (Kc) variou em função do estádio de desenvolvimento da cultura, adotando-se os valores de 0,3, 0,70 , 1,0 , 0,95 e 0,90 para os diferentes estádios, de acordo com Doorenbos \& Kassam (1979).

Os dados para a obtenção das curvas de avanço e infiltração da água no solo foram obtidos em cinco ensaios conduzidos antes da semeadura. Na execução dos ensaios de infiltração foram medidas as vazões de entrada e de saída de água nos sulcos, utilizando-se calhas WSC, apropriadas para vazões de 0,07 a $2,3 \mathrm{~L} \mathrm{~s}^{-1}$.

A equação utilizada para descrever o avanço da água foi a seguinte (Willardson \& Bishop, 1967):

$$
T_{a v}=a\left(e^{b x}-1\right)
$$

em que:

$\mathrm{T}_{\mathrm{av}}$ - tempo de avanço, em min

$\mathrm{x}$ - distância do avanço, em $\mathrm{m}$

a, b - coeficientes de ajuste

O processo de infiltração da água no solo foi representado pela equação proposta por Kostiakov, citados por Walker \& Skogerboe (1987) ou seja:

$$
I=K T^{n}
$$

sendo:

I - intensidade de infiltração, em $\mathrm{mm} \mathrm{h}^{-1}$

$\mathrm{K}$ - constante relativa à taxa de infiltração na unidade de tempo

$\mathrm{T}$ - tempo de infiltração, em min

n - coeficiente de ajuste

Para cada tratamento, que corresponde à aplicação de determinada lâmina de água no sulco, procedeu-se a avaliação da irrigação, determinando-se as eficiências de aplicação, de armazenamento, de distribuição e as perdas por percolação e por escoamento superficial, segundo Bernardo (1989).

No experimento foi plantada a cultura do feijão (Phaseolus vulgaris, L) cultivar Aroana 80, tipo arbustivo, com guia curta e ciclo total de 90 a 100 dias. Procurou-se obter em torno de 200.000 plantas ha ${ }^{-1}$, o que corresponde a 10 plantas $\mathrm{m}^{-1}$, no espaçamento entre linhas de 1,0 m. As adubações fosfatada e nitrogenada foram feitas com base na análise de solo, utilizando-se superfosfato na quantidade de $400 \mathrm{~kg} \mathrm{ha}^{-1}$ e sulfato de amônio, na quantidade de $100 \mathrm{~kg} \mathrm{ha}^{-1}$.

Para avaliação de rendimento da cultura foram considerados os seguintes parâmetros: produtividade de grãos, número de vagens por planta, número de grãos por vagem e matéria seca da parte aérea. Procedeu-se a uma análise estatística desses parâmetros, considerando-se as posições relativas ao início, meio e final do sulco.

\section{RESULTADOS E DISCUSSÃO}

\section{Avanço e infiltração da água}

Os tempos de avanço para a água percorrer os $120 \mathrm{~m}$ de sulco, para as vazões de 1,0 e $0,35 \mathrm{~L} \mathrm{~s}^{-1}$, foram de 33 e 155,5 min, respectivamente. Na Figura 1 estão apresentadas as curvas e as equações de avanço obtidas na primeira irrigação.

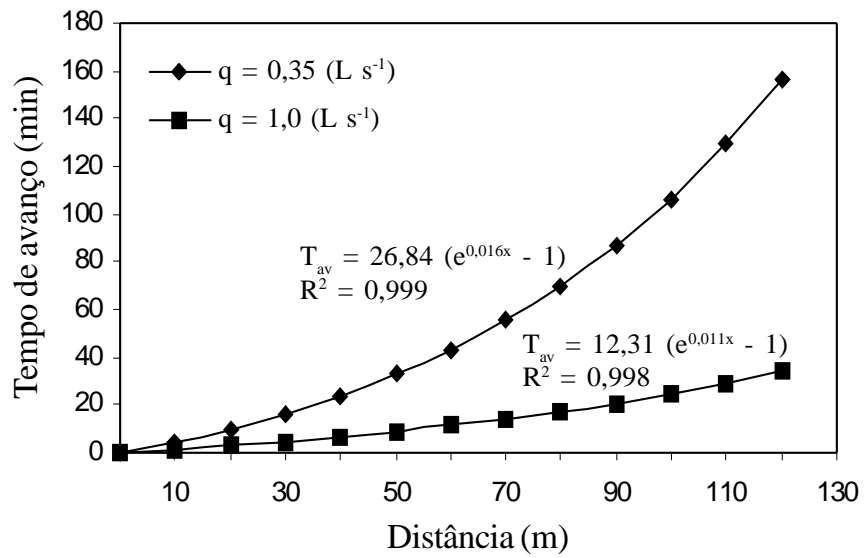

Figura 1. Curvas e equações de avanço da água no sulco, na primeira irrigação

Para a aplicação da lâmina útil de $21 \mathrm{~mm}$, necessária para a elevação da umidade do solo à capacidade de campo, os tempos de irrigação para os tratamentos $\mathrm{T}_{1}, \mathrm{~T}_{2}$ e $\mathrm{T}_{3}$ foram de 182,07, 100,2 e 155,5 min, respectivamente.

As curvas de velocidade de infiltração obtidas na primeira irrigação estão apresentadas na Figura 2. Comparando-se as velocidades de infiltração básica, observa-se que estas diminuíram com a diminuição das vazões.

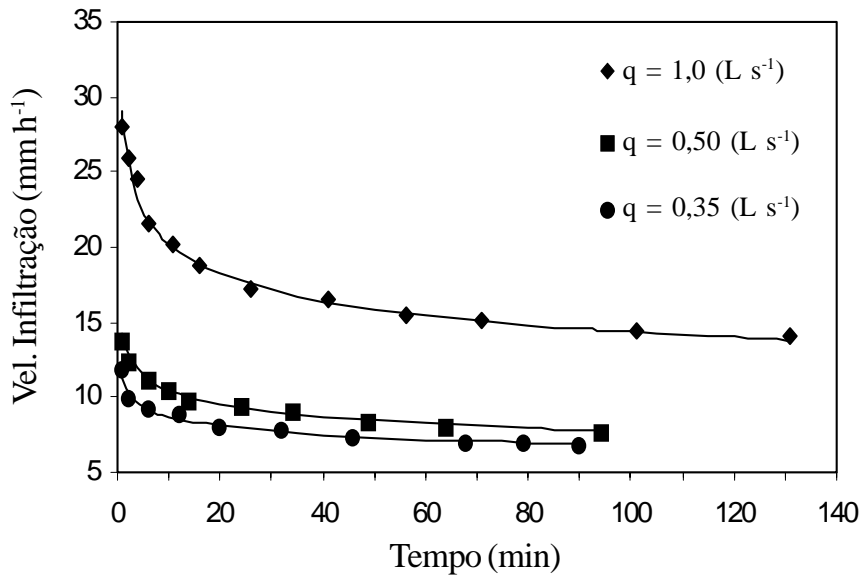

Figura 2. Velocidade de infiltração da água no solo, na primeira irrigação

\section{Manejo da irrigação}

As eficiências de aplicação, armazenamento e distribuição e as perdas por percolação e escoamento superficial, para os tratamentos, estimadas a partir das equações de avanço e infiltração da água no sulco, estão apresentadas na Tabela 1. De 
acordo com Bernardo (1989) e em condições ideais de manejo de irrigação, circunstância considerada no tratamento $T_{1}$, os valores aceitáveis de eficiência de distribuição e de aplicação são de 70 e 60\%, respectivamente. A eficiência de aplicação de $39,1 \%$ obtida indica que a irrigação deveria ser conduzida com outras reduções de vazão.

Comparando-se os tratamentos $\mathrm{T}_{1}$ e $\mathrm{T}_{2}$ observa-se que, com a redução do tempo de irrigação de 182,07 para 100,2 min, há uma redução na eficiência de armazenamento de 100 para 80,9\% e na eficiência de distribuição de 81,9 para $68,5 \%$ (Tabela 1); no entanto, ocorre aumento na eficiência de aplicação, o que representa uma economia de $38 \%$ de água no tratamento $\mathrm{T}_{2} \mathrm{em}$ relação ao tratamento $\mathrm{T}_{1}$, evidenciada pelas perdas totais de água por percolação e escoamento superficial.

Tabela 1. Valores médios de eficiência de armazenamento (Es), eficiência de aplicação (Ea), eficiência de distribuição (Ed) e perdas por percolação $(\mathrm{Pp})$ e por escoamento superficial $(\mathrm{Pe})$ nos diferentes tratamentos, em \%

\begin{tabular}{crrrrr}
\hline Tratamento & \multicolumn{1}{c}{ Es } & \multicolumn{1}{c}{ Ea } & \multicolumn{1}{c}{ Ed } & Pp & \multicolumn{1}{c}{ Pe } \\
\hline $\mathrm{T}_{1}$ & 100,0 & 39,1 & 81,9 & 8,6 & 52,3 \\
$\mathrm{~T}_{2}$ & 80,9 & 51,4 & 68,5 & 0,0 & 48,6 \\
$\mathrm{~T}_{3}$ & 66,9 & 100,0 & 0,0 & 0,0 & 0,0 \\
\hline
\end{tabular}

No tratamento $T_{3}$ verifica-se a pior condição para a cultura, com baixa eficiência de armazenamento e lâmina de água deficiente em todo o comprimento do sulco, embora a eficiência de aplicação seja de $100 \%$ e as perdas nulas.

A eficiência de armazenamento verificada no tratamento $\mathrm{T}_{3}$ indica a ocorrência de déficit hídrico e, conseqüentemente, o mesmo ocorreu no tratamento $\mathrm{T}_{4}$ (testemunha) provocando atraso na formação de vagens com relação aos tratamentos $\mathrm{T}_{1} \mathrm{e}$ $\mathrm{T}_{2}$, fato este que está de acordo com MacMaster et al. (1965) apud Salter \& Goode (1967) que observaram redução do ciclo da cultura nos tratamentos que receberam umidade adequada, em relação aos tratamentos com déficit, e também observado por Bergamaschi (1984) no tratamento com deficiência de água durante a formação dos botões florais e na floração.

Nos tratamentos $\mathrm{T}_{1}$ e $\mathrm{T}_{2}$ a cultura esteve, em maior parte do seu ciclo, sob tensões de água no solo inferiores a - $80 \mathrm{kPa}$ e nos tratamentos $\mathrm{T}_{3}$ e $\mathrm{T}_{4}$ sob tensões superiores. Este fato está relacionado com a lâmina de água aplicada nos tratamentos, influenciando a umidade na região radicular das plantas.

\section{Rendimento da cultura}

Pela análise de variância da produtividade de grãos, verificou-se que o efeito dos tratamentos sobre a produtividade foi altamente significativo a nível de $1 \%$ de probabilidade e o efeito da interação tratamento versus posição no sulco não foi significativo.

Comparando-se as médias da produtividade de grãos da Tabela 2, observa-se que os tratamentos $\mathrm{T}_{1}, \mathrm{~T}_{2} \mathrm{e}_{3}$ apresentaram aumento de produtividade de 70,1,65,4 e 34,6\%, em relação à testemunha $\left(\mathrm{T}_{4}\right)$. As baixas produtividades dos tratamentos $\mathrm{T}_{3}$ e $\mathrm{T}_{4}$ podem estar relacionadas ao estresse hídrico nos períodos de preflorescimento e florescimento, devido a irrigação deficiente, fato este evidenciado por vários pesquisadores (Magalhães \& Millar, 1978; Gunton \& Evenson, 1982; Sawazaki \& Teixeira, 1981). Os maiores rendimentos ocorreram nos
Tabela 2. Produtividade média de grãos*, em kg ha ${ }^{-1}$, no início, meio e final do sulco, para os diferentes tratamentos

\begin{tabular}{crrrc}
\hline Tratamento & Início & \multicolumn{1}{c}{ Meio } & \multicolumn{1}{c}{ Final } & Média \\
\hline $\mathrm{T}_{1}$ & 1548,9 & 1522,8 & 1307,8 & $1459,8 \mathrm{a}$ \\
$\mathrm{T}_{2}$ & 1384,8 & 1562,7 & 1312,3 & $1419,9 \mathrm{a}$ \\
$\mathrm{T}_{3}$ & 1144,2 & 1268,0 & 1054,1 & $1155,4 \mathrm{ab}$ \\
$\mathrm{T}_{4}$ & 905,1 & 921,6 & 747,8 & $858,2 \mathrm{~b}$ \\
\hline *Mér.
\end{tabular}

(Tukey $1 \%$ ) 356,6; DMS dentro dos tratamentos (Tukey $1 \%$ ) $=480,3$

tratamentos $\mathrm{T}_{1}$ e $\mathrm{T}_{2}$ e o menor no tratamento $\mathrm{T}_{4}$ e as médias dos tratamentos $\mathrm{T}_{1}$ e $\mathrm{T}_{2}$ diferiram estatisticamente do tratamento $\mathrm{T}_{4}$.

\section{Número de vagens por planta}

Pela análise de variância do número de vagens por planta, verificou-se que o efeito dos tratamentos foi significativo a nível de $5 \%$ de probabilidade, enquanto o efeito da interação tratamento versus posição no sulco foi significativo a nível de $5 \%$ no tratamento $\mathrm{T}_{3}$.

Na Tabela 3 são apresentados os valores médios referentes ao número de vagens por planta, obtidos nas posições início, meio e final do sulco, nos diferentes tratamentos. O maior número médio de vagens por planta ocorreu nos tratamentos $\mathrm{T}_{1}$ e $\mathrm{T}_{2}$ e o menor no tratamento $\mathrm{T}_{4}$; as médias dos tratamentos $\mathrm{T}_{1} \mathrm{e}_{2}$ diferiram estatisticamente do tratamento $\mathrm{T}_{4}$.

Tabela 3. Número médio de vagens*, no início, meio e final do sulco, para os diferentes tratamentos

\begin{tabular}{ccccc}
\hline Tratamento & Início & Meio & Final & Média \\
\hline $\mathrm{T}_{1}$ & 11,5 & 12,5 & 12,6 & $12,2 \mathrm{a}$ \\
$\mathrm{T}_{2}$ & 14,5 & 13,6 & 10,1 & $12,7 \mathrm{a}$ \\
$\mathrm{T}_{3}$ & 9,6 & 12,5 & 7,7 & $9,5 \mathrm{ab}$ \\
$\mathrm{T}_{4}$ & 7,7 & 10,3 & 7,4 & $8,5 \mathrm{~b}$ \\
\hline
\end{tabular}

* Médias seguidas da mesma letra não diferem estatisticamente; DMS para tratamentos (Tukey $1 \%$ ) 3,6; DMS dentro dos tratamentos (Tukey $1 \%$ ) $=4,6$

Observou-se decréscimo do número de vagens por planta do meio para o final do sulco, nos tratamentos $\mathrm{T}_{2}, \mathrm{~T}_{3}$ e $\mathrm{T}_{4}$.

\section{Número de grãos por vagem}

Pela análise de variância, os efeitos dos tratamentos foram altamente significativos a nível de $1 \%$ de probabilidade, mas o efeito da interação tratamento versus posição no sulco não foi significativo.

Na Tabela 4 são apresentados os valores médios referentes ao número de grãos por vagem obtidos nas posições início, meio e final do sulco, nos diferentes tratamentos.

Tabela 4. Número médio de grãos por vagem*, no início, meio e final do sulco, para os diferentes tratamentos

\begin{tabular}{ccccc}
\hline Tratamento & Início & Meio & Final & Média \\
\hline $\mathrm{T}_{1}$ & 4,2 & 4,5 & 4,0 & $4,3 \mathrm{a}$ \\
$\mathrm{T}_{2}$ & 4,4 & 4,3 & 4,3 & $4,3 \mathrm{a}$ \\
$\mathrm{T}_{3}$ & 4,3 & 4,2 & 3,8 & $4,1 \mathrm{ab}$ \\
$\mathrm{T}_{4}$ & 3,8 & 3,4 & 3,7 & $3,6 \mathrm{~b}$ \\
\hline
\end{tabular}

* Médias seguidas da mesma letra não diferem estatisticamente; DMS para tratamentos (Tukey $1 \%$ ) 0,5 ; DMS dentro dos tratamentos (Tukey $1 \%$ ) $=0,6$

Comparando-se as médias, observa-se maior número de grãos por vagem nos tratamentos $\mathrm{T}_{1}$ e $\mathrm{T}_{2}$ e menor no tratamento $\mathrm{T}_{4}$; as médias referentes aos tratamentos $\mathrm{T}_{1}$ e $\mathrm{T}_{2}$ diferem estatisticamente da média do tratamento $\mathrm{T}_{4}$. 
Matéria seca da parte aérea

Pela análise de variância da matéria seca total, verificou-se que o efeito de tratamentos foi significativo a nível de $1 \%$ de probabilidade, mas a interação tratamento versus posição no sulco não foi significativa.

Na Tabela 5 estão apresentados os valores médios referentes à matéria seca da parte aérea, obtidos nas posições início, meio e final do sulco, nos diferentes tratamentos. Comparando-se as médias, observa-se que o peso de matéria seca total da parte aérea nos tratamentos $\mathrm{T}_{1}$ e $\mathrm{T}_{2}$ diferiu estatisticamente do peso do tratamento $\mathrm{T}_{4}$.

Tabela 5. Matéria seca*, em grama por 10 plantas, da parte aérea do feijoeiro no início, meio e final do sulco, para os diferentes tratamentos

\begin{tabular}{crrrc}
\hline Tratamento & Início & Meio & Final & Média \\
\hline $\mathrm{T}_{1}$ & 130,3 & 150,0 & 132,5 & $137,6 \mathrm{a}$ \\
$\mathrm{T}_{2}$ & 158,1 & 156,7 & 112,0 & $142,3 \mathrm{a}$ \\
$\mathrm{T}_{3}$ & 105,7 & 137,4 & 77,6 & $106,9 \mathrm{ab}$ \\
$\mathrm{T}_{4}$ & 78,7 & 95,5 & 70,9 & $81,7 \mathrm{~b}$ \\
\hline
\end{tabular}

* Médias seguidas da mesma letra não diferem estatisticamente; DMS para tratamentos (Tukey 1\%) = 47,$0 ;$ DMS dentro dos tratamentos (Tukey $1 \%$ ) $=67,1$

O efeito do déficit hídrico acarreta redução na matéria seca total, traduzida por decréscimo no índice de crescimento, cuja observação no presente trabalho está de acordo com diversos autores (Slayter, 1969; Millar \& Gardner, 1972 ; Begg \& Turner, 1976).

\section{CONCLUSÕES}

1. A aplicação de $50 \%$ da lâmina de água útil no final da parcela não alterou significativamente a produtividade do feijoeiro.

2. A deficiência de água provocou atraso na formação de vagens e decréscimo no número de grãos por vagem.

3. A diminuição da lâmina de água do final para o início do sulco, resultou em menores valores dos parâmetros de rendimento, devido ao estresse gradual nos tratamentos de $0 \%$ e 50\%, da lâmina útil aplicada.

\section{REFERÊNCIAS BIBLIOGRÁFICAS}

AGRIANUAL-96. FNP Consultoria. São Paulo, 1996. 249p.

BASCUR, B.G.; FRITSCH, F.N. Efectos de metodos y frequencia de riego sobre componentes del rendimiento en frijol (Phaseolus vulgaris, L). Agricultura Técnica. Chile, v.35,n.3,p.147-52, 1975.

BEGG, J.E.; TURNER, N.C. Crop water deficits. Advances in Agronomy. San Diego, v.28, p.161-217, 1976.

BERGAMASCHI, H. Perda de água e desenvolvimento do feijoeiro (Phaseolus vulgaris $\mathrm{L}$ ) sob diferentes condições de disponibilidade hídrica no solo e demanda evaporativa. Piracicaba, ESALQ/USP, 1984. 204p. Tese Doutorado

BERNARDO, S. Manual de irrigação. 2. ed., Viçosa, Imprensa Universitária, 1989.463p.

DOORENBOS, J.; KASSSAM, A.H. Crop response to water. Rome: FAO, 1979. 194p. Irrigation and Drainage Paper, n.33.
DOORENBOS, J.; PRUITT, W.O. Guidelines for predicting crop water requirements. Rome: FAO, 1975. 179p. Irrigation and Drainage Paper, n.24.

FISCHER, V.J.; WEAVER, C.K. Flower, pod set, and pod retention of Lima Bean in response to night temperature, humidity and soil moisture. Journal of American Society Horticulture Science. Alexandria, v.99, p.448-450. 1974.

FRIZZONE, J.A. Funções de resposta do feijoeiro (Phaseolus vulgaris, L) ao uso de nitrogênio e lâmina de irrigação. Piracicaba, ESALQ/USP, 1986. 133p. Tese Doutorado

FRIZZONE, J.A. Irrigação por superfície: Princípios de operação e manejo. Piracicaba: ESALQ/USP - Departamento de Engenharia Rural, 1992. 82p. Série Didática 004

GARRIDO, M.A.T.; TEIXEIRA, H.A. Efeito de diferentes níveis de umidade do solo sobre o rendimento do feijoeiro na região sul de Minas Gerais. Belo Horizonte: EPAMIG, 1978. 65p. Projeto Feijão, Relatório 75/76.

GUIMARÃES, C.N. Efeitos fisiológicos do estresse hídrico. In.: ZIMERMANN, M.J. de O.; ROCHA, M.; YAMADA, T. ed. Cultura do feijoeiro: Fatores que afetam a produtividade. Piracicaba: Associação Brasileira para Pesquisa da Potassa e do Fosfato, 1988. p.157-174.

GUNTON, J.L.; EVENSON, J.P. Moisture stress in Navy Beans. Effect of withholding irrigation at different phenological stage on growth and yield. Irrigation Science, Berlin, v.2, n.1, p.4958. 1982.

HOSTALÁCIO, S.; VÁLIO, I.F.M. Desenvolvimento de plantas de feijão cv. Goiano Precoce, em diferentes regimes de irrigação. Pesquisa Agropecuária Brasileira, Brasilía, v.19, p.211-218, 1988.

MAGALHÃES, A.A.; MILLAR, A.A. Efeito do déficit de água no período reprodutivo sobre a produção do feijão. Pesquisa Agropecuária Brasileira. Brasília, v.13, n.2, p.55-60, 1978.

MAGALHÃES, A.A.; MILLAR, A.A.; CHOUDHURY, E.N. Efeito do déficit fenológico de água sobre a produção de feijão. Turrialba. Turrialba, v.29,n.4,p.269-273. 1979.

MILLAR, A.A.; CHOUDHURY, E.N. A model to define operation irrigation frequency for maximum yield of crop. Turrialba, Turrialba, v.30, n.4, p.391-398, 1980.

MILLAR, A.A.; GARDNER, W.R. Effect of the soil water potential on the dry matter production of snap beans. Agronomy Journal. Madison, v.64, n.5, p.559-562,1972.

POZZEBON, E.J. Resposta da cultura do feijão à irrigação, fertirrigação e uso de micronutrientes. Santa Maria: Universidade Federal de Santa Maria. 1995. 127p. Dissertação Mestrado

SALTER, P.J.; GOODE, J.C. Crop responses to water at different stages of growth. Bucks: Commonwealth Agricultural Bureaux. 1967. p.52-57.

SAWAZAKI, H.E.; TEIXEIRA, J.P.F. Estresse de água no crescimento, produtividade e acúmulo de prolina em feijão Bragantia, Campinas, v.40, p.157-166, 1981.

WALKER, W.R.; SKOGERBOE, G.V.Surface irrigation: Theory and practice. New Jersey: Englewood Cliffs, Prentice Hall, 1987.386p.

WILLARDSON, L.S.; BISHOP, A.A. Analysis of surface irrigation application efficiency. Journal of Irrigation and Drainage Division. ASCE, New York, v.93, n.2, p.21-36. 1967. 